\title{
21 PROXIMAL BUT NOT DISTAL AORTIC STIFFNESS EXPLAINS BLOOD PRESSURE REDUCTION ASSOCIATED WITH EXERCISE TRAINING FOR A FIRST TIME MARATHON
}

Anish N Bhuva ${ }^{1,2}$, Andrew D'Silva ${ }^{3}$, Camilla Torlasco ${ }^{2,4}$, Niro Nadarajan', Siana Jones ${ }^{1}$, Kristopher D Knott' ${ }^{1,2}$, Giulia Benedetti ${ }^{2}$, Paul Scully ${ }^{1,2}$, Rachael Bastiaenen ${ }^{3}$, Peter Kellman ${ }^{5}$, Guy Lloyd ${ }^{2}$, Nishi Chaturvedi ${ }^{1}$, Sanjay Sharma ${ }^{3}$, James C Moon ${ }^{1,2}$, Alun D Hughes $^{1}$, Charlotte H Manisty ${ }^{1,2}$

${ }^{1}$ University College London, UK; ${ }^{2}$ Barts Heart Centre, London, UK; ${ }^{3}$ St. George's University, London, UK; ${ }^{4}$ Istituto Auxologico Italiano, Milan, Italy; ${ }^{5}$ National Institutes of Health, USA.

Introduction Exercise training has beneficial effects on blood pressure (BP), however the physiological mechanisms remain unclear, and the impact on regional aortic haemodynamics is unexplored. We studied the effects of exercise training on central BP (cBP) and regional aortic stiffness using cardiovascular magnetic resonance (CMR).

Methods Untrained healthy volunteers were recruited prior to starting an exercise training programme for the London Marathon. Assessment pre-training and up to three weeks post-marathon included cBP; anthropometry, maximum oxygen consumption (VO2max) and CMR. Phase contrast imaging $(1.97 \times 1.77 \mathrm{~mm} 2,9.24 \mathrm{~ms}$ temporal resolution) at the pulmonary artery and diaphragm levels was used to calculate regional aortic distensibility and pulse wave velocity (PWV). Data are means (95\% confidence intervals).

Results 136 marathon finishers (median age 34 (range 21-69 years), 49\% male) were studied. Following exercise training, cBP reduced by $4.2(2.6,5.9) / 2.6(1.6,3.5)$ $\mathrm{mmHg}$ and heart rate by $2.4(0.3,4.3)$ min-1. Total aortic PWV reduced by -0.21 ($0.01,-0.42) \mathrm{m} / \mathrm{s}$ and proximal descending aortic distensibility increased by $0.83(0.2$, 1.46) 10-3 mmHg-1. Older age and higher baseline BP (but not change in weight or VO2max) were associated with greater reduction in cBP. Reduction in cBP was correlated with PWV reduction (or increased distensibility) in the proximal but not the distal aorta $(r=0.18(0.02,0.32)$ and $0.03(-0.14,0.20)$ respectively).

Conclusion Exercise training results in a reduction in central blood pressure; which can be explained, at least partially, by reduction in aortic stiffness in the proximal aorta. This benefit appears greatest in older subjects and those with higher baseline blood pressures. 